\title{
Liquid biopsies and cancer omics
}

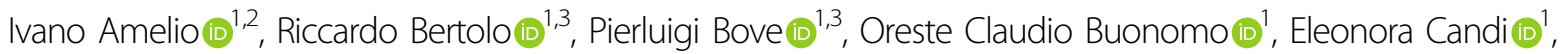

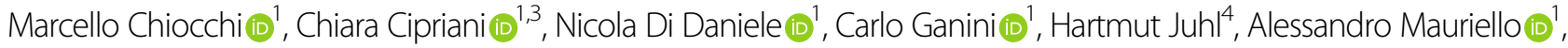

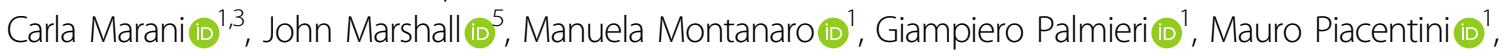

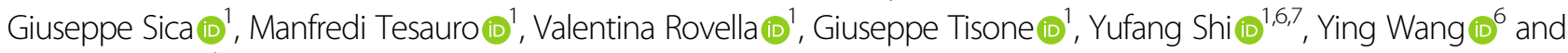 \\ Gerry Melino (1) ${ }^{1}$
}

\begin{abstract}
The development of the sequencing technologies allowed the generation of huge amounts of molecular data from a single cancer specimen, allowing the clinical oncology to enter the era of the precision medicine. This massive amount of data is highlighting new details on cancer pathogenesis but still relies on tissue biopsies, which are unable to capture the dynamic nature of cancer through its evolution. This assumption led to the exploration of non-tissue sources of tumoral material opening the field of liquid biopsies. Blood, together with body fluids such as urines, or stool, from cancer patients, are analyzed applying the techniques used for the generation of omics data. With blood, this approach would allow to take into account tumor heterogeneity (since the circulating components such as CTCS, ctDNA, or ECVs derive from each cancer clone) in a time dependent manner, resulting in a somehow "real-time" understanding of cancer evolution. Liquid biopsies are beginning nowdays to be applied in many cancer contexts and are at the basis of many clinical trials in oncology.
\end{abstract}

\section{Facts}

- Oncology has entered the era of Precision Medicine. we still however struggle in capturing the dynamic nature of cancer.

- Liquid biopsies represent a valuable source of information on each individual cancer and can be used to monitor cancer evolution.

- Components of liquid biopsies (CTCs, ctDNA, ctRNA and ECVs) have been proven to correlate with cancer prognosis and cancer biology in many clinical cancer entities (such as breast cancer or neuroblastoma).

\footnotetext{
Correspondence: Ivano Amelio (ivano.amelio@uniroma2.it) or Gerry Melino (melino@uniroma2.it)

${ }^{1}$ Torvergata Oncoscience Research Centre of Excellence, TOR, Department of Experimental Medicine, University of Rome Tor Vergata, 00133 Rome, Italy

${ }^{2}$ School of Life Sciences, University of Nottingham, Nottingham, UK

Full list of author information is available at the end of the article

Edited by R.A. Knight
}

\section{Open questions}

- Are liquid biopsies sufficient to capture tumoral evolution?

- How can we discriminate the cancer subclonesderived components of liquid biopsies?

- Will liquid biopsies ever overcome tissue biopsies in the clinical management of cancer?

\section{Background}

Clinical oncology has been relying on the increasing amount of molecular data which can be obtained from single cancer specimens ${ }^{1}$. The molecular profiling of gene mutations for prognostic predictions or for therapy selection can be accounted as a standard approach in many cancer entities since decades. The scientific community moved from a purely histopathologic cancer diagnosis to a molecular-based one, allowing clinicians to develop more accurate and complex prognostic scores, as well as to select better treatment options according to the mutational background of a given 


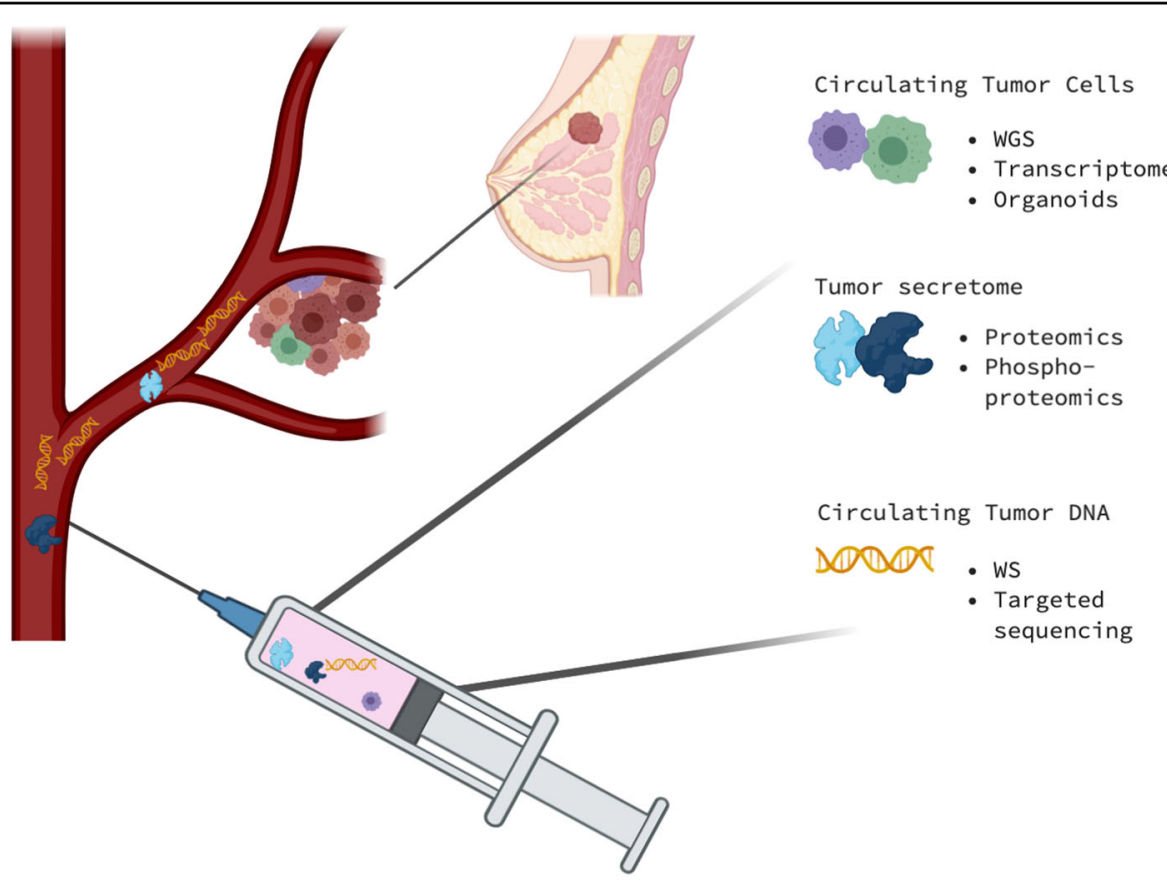

Fig. 1 The multiomics approach on liquid biopsies. The information collected from a single blood specimen can reflect the evolution of a single cancer from many biological points of view. Circulating tumor cells (CTCS) highly reflect the complexity of the pathology, especially regarding tumor heterogeneity. Full genomic (whole genome sequencing) and transcriptomic analysis can be applied to CTCs and can be used as well for the growth of cancer organoids. Circulating proteins can also reflect the tumor secretome and can be analyzed through spectrometric approaches such as proteomics and phosho-proteomics. Moreover, circulating tumor DNA can be purified and used for whole or targeted sequencing. Picture created with Biorender.com.

neoplasm (as in the case of EGFR or ALK mutations in lung cancer ${ }^{2}$, or the V600E mutation of BRAF in either colorectal cancer $^{3}$ or melanoma, as prominent examples). This approach opened the era of targeted therapies and, to some extent, to precision medicine $\mathrm{e}^{4-6}$. "Intelligent drugs" designed to specifically target a precise molecular objective, have entered the clinics alongside classic chemotherapy or substituting it ${ }^{7}$. Although this new options generated great therapeutical advances in many cancer contexts (such as the VEGF/VEGFR targeting in kidney cancer ${ }^{8}$ or in hepatocellular carcinoma, or the anti-BCR/Abl rearrangement for the therapy of chronic monocytic leukemia ${ }^{9}$ ) they still often result in a time limited controlled status of the disease, invariably leading to cancer progression in most cases, without considering primary refractories cancer entities, which are not responsive to given targeted therapies since the beginning ${ }^{10}$. Sometimes, this inevitable failure is partially due to the heterogeneous nature of cancers and to the evolutive pressure that is mediated by cancer treatment itself. Tumor biopsies can give limited information on a single cancer entity, since they cannot account for its intrinsic tumor heterogeneity and, moreover, for its evolution during time, due to their invasive nature which impairs the possibility of their repetition to follow cancer evolution ${ }^{11-15}$.

Great advances in the sequencing technologies are at the roots of the generation of many more accurate molecular data from single cancer specimens. Whole cancer genomes analysis can now be performed at relatively low costs, together with many other "omics", such as whole transcriptomic or proteomic (which can also be further completed by phospho-proteomics, giving a wide picture on the activation of signaling pathways $)^{15-24}$. These techniques led to the generation of vast amounts of data derived from a single cancer specimen (a diagnostic biopsy or a surgical removal of the disease), therefore determining a molecular deep characterization of a single cancer in a precise and limited time of the disease.

Cancer specimens are not the most ideal source material for capturing the dynamic nature of cancers, since they cannot be repeated during the evolution of the disease due to their invasive nature. Anyway, tissues like blood, have been shown to harbor many biological entities which directly derive from cancer itself and can be exploited as the ideal source for liquid biopsies (Fig. 1) $)^{25-28}$.

\section{Liquid biopsies in cancer}

The circulating blood reflects primary and metastatic tumor biology, since tumor cells are more prone than normal cells to release nucleic acids into the bloodstream upon death. Circulating tumor DNA (ctDNA) and well as circulating tumor RNA (ctRNA) can be purified from the plasma of cancer patients, and analyzed through next 
generation sequencing or targeted sequencing. Both normal and tumoral DNA are circulating and cannot be properly separated form each other but the most abundant source of cell-free DNA is the tumoral one (a mass of $100 \mathrm{~g}$ of tumor burden is estimated to release $3.3 \%$ of its DNA content) with a 10-100-fold abundancy compared to the normal $\mathrm{DNA}^{29}$.

Liquid biopsies can also rely on other aspects of cancer tumorigenesis such as on circulating tumor cells (CTCs) and extra-cellular vesicles $(\mathrm{ECVs})^{30}$. CTCs can be isolated from peripheral blood and discriminated form normal cells using anti-EpCAM or anti-CK or CD45 selection methods. They are extremely rare ( $<10$ cells $/ \mathrm{ml}$ of blood) and have been isolated from almost all human cancers. CTCs correlate with prognosis and with a metastatic disease status and their clinical role in defining cancer prognosis has been stated by the FDA approval for their detection in breast cancer and prostate cancer ${ }^{31,32}$. CTCs account for tumor heterogeneity since the circulating cells reflect cancer subclones and can be the ideal source for whole genome sequencing and transcriptomic analysis. Moreover, they can also be used to establish organoids cultures, which are proven to be a valuable in vitro reproduction of an individual cancer ${ }^{33}$.

Moving from cells to smaller biological entities, ECVs can be separated from plasma through different methodologies (size exclusion chromatography, affinity purification, and differential ultracentrifugation). As compared to the proteomic profiling of circulating proteins, ECVs show highly enriched exosome proteins and therefore constitute a valuable source of information from a single cancer. Proteins are not the only components of ECVs since tumor DNA and RNA (especially micro-RNA) are also present. Moreover, they are not solely inert tumoral material, since they can function as signal transductor and are highly present in the tumor microenvironment, where they can signal through an autocrine/ paracrine pathway or be released in the bloodstream for long distance signaling ${ }^{34,35}$.

Blood is surely the most characterized tissue available for liquid biopsies while many other corporeal fluids are suitable for the detection of tumoral components. Saliva, urines, seminal fluids, tears, and stool have been analyzed to understand their correlation with cancer ${ }^{36}$. Stool in particular have been thoroughly evaluated in the context of gastrointestinal cancers, and proved to be a valuable source of tumoral DNA for pancreatic cancer ${ }^{37-40}$. Stool analysis from a multiomics perspective is also able to integrate information on the role of the microbiome in cancer pathogenesis, especially in the case of colorectal cancer and pancreatic cancer, as well as in other disease entities such as inflammatory intestinal diseases ${ }^{41-43}$. The microbiota creates a complex network that can influence the tumor microenvironment in a very heterogeneous way that relies on the intrinsically heterogeneity of the microbiome itself $^{44-49}$. The microbiome study, also through liquid biopsies, would also grant some other information related to the geography of diseases, among which cancer, since the intestinal bacteria are able to differentiate individuals on the basis of the place they live $i^{50-54}$.

The main advantage of a liquid biopsy approach basically stands with the possibility to capture tumor heterogeneity in a whole (all the tumor subclones release CTCs and ctDNA in the bloodstream) through its evolution, since they are totally not harmful for the patients. The combination of this approach, together with the study of the related omics, has led to promising results in the oncology world.

This review will focus on two disease entities, breast cancer and neuroblastoma, to highlight the state of art in the field.

\section{Liquid biopsies for breast cancer}

Breast cancer represent a striking evidence for the advantage of liquid biopsies use in the everyday medical treatment. The Cellsearch ${ }^{\circledR}$ test has been approved by FDA for the extraction and selection of CTCs from breast cancer patients (but also for prostate cancer) for the determination of their prognosis (Fig. 2) ${ }^{55,56}$.

CTCs arise during early stages of breast cancer as shown on triple negative breast cancer patients (TBNC), where this cell population at diagnosis is quite heterogeneous in terms of expression of hormone receptors (HR), HER2, or EGFR (varying from 24.4\% of expression of HR, 20\% for HER2 and $40 \%$ for EGFR) while only the HER2 positive CTCs prevail after adjuvant treatment ${ }^{57}$. Deeper understanding on breast cancer CTCs comes from the evidence that this cell population expresses both epithelial (keratins, EpCAM, and cadherin 1) and mesenchymal markers (cadherin 2, fibronectin, and serpin peptidase inhibitor), suggesting that they might be subject to the epithelial-to-mesenchymal (EMT) reprogramming. Anyway, the most represented subgroup of CTCs expresses mesenchymal markers therefore confirming that EMT plays a pivotal role in their generation ${ }^{58}$. This is highly remarkable considering that CTCs in breast cancer correlate with metastasis development, and that EMT is highly associated to the development of a metastatic disease from a clinical point of view ${ }^{59}$. CTCs do represent a causative biologic event for the insurgence of metastasis also in the context of brain metastasis, where they show a distinctive "breast cancer brain metastasis gene signature" and can be utilized as predictive biomarkers of this clinical event ${ }^{60}$.

As abovementioned, a liquid biopsy does not only rely on the evaluation of CTCs. The secretion of proteins from tumor cells has been investigated through label-free quantitative proteomics approaches on plasma samples. 


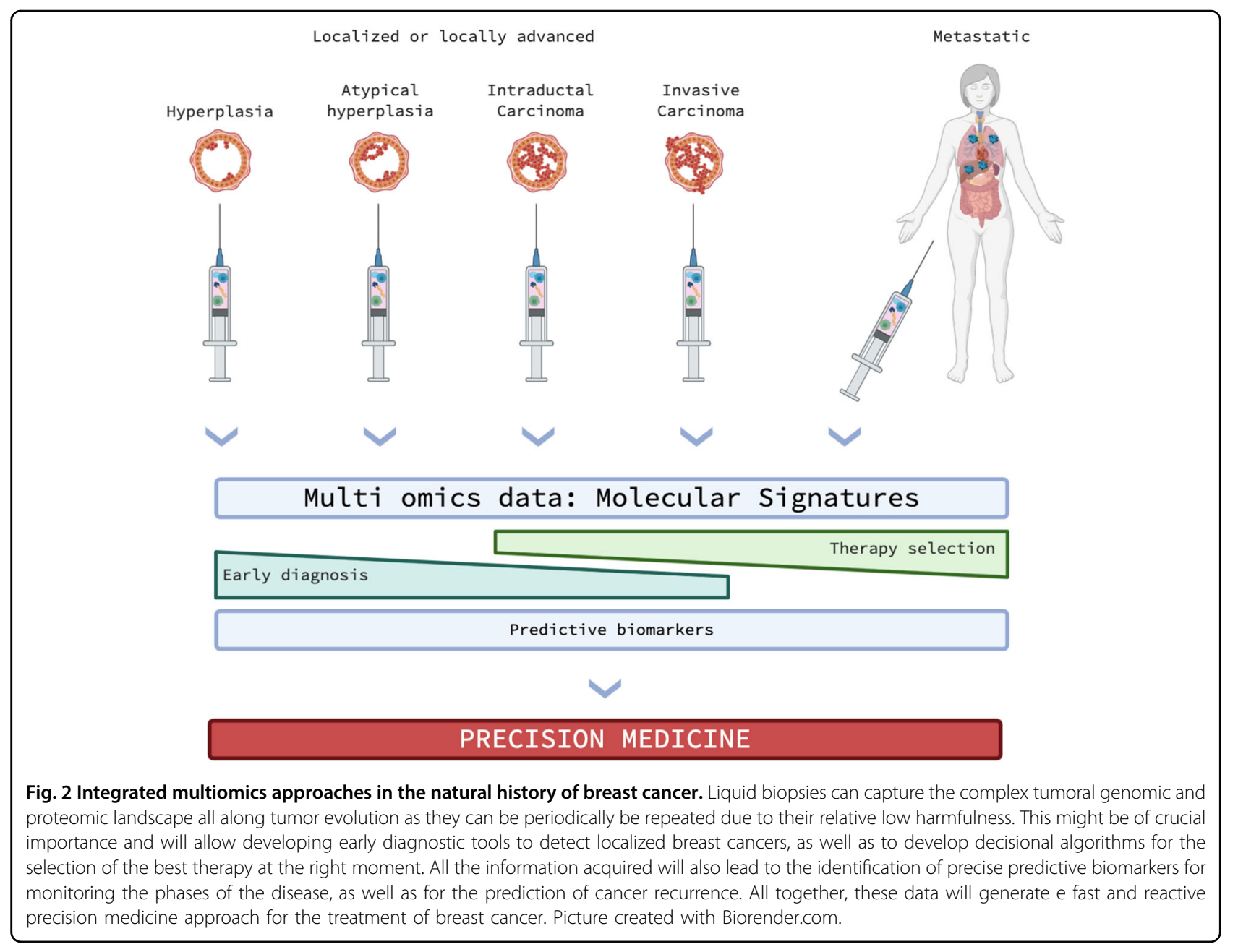

Tumoral secretomes are highly abundant in plasma since cancer cells are much more prone to shedding and releasing their content into the bloodstream, as compared to healthy cells. The study of secreted proteins through liquid chromatography tandem mass spectrometry combined to RNA sequencing approaches, has been proven to be able to distinguish among breast cancer phenotypes with different prognosis, and proved to be a valuable strategy for outcome prediction ${ }^{61-64}$. Proteogenomics approaches such as the one described were further validated in a larger study conducted on 105 annotated breast cancer genomes, allowing to describe some genomic alterations through a more mechanistic perspective. This is the case of the loss of $5 \mathrm{q}$ chromosome, which is a frequent finding in basal-like breast carcinomas, that led to the identification of the loss of CETN3 and SPK1 correlating with higher expression of EGFR ${ }^{65}$.

Proteins are not only secreted in the bloodstream, they can also be incorporated within tumor-derived extracellular vesicles, together with other components such as DNA or RNA. In breast cancer, the role of ECVs has been explored in a mouse model, showing that ECVs target bone marrow CD11+ cells, impairing their differentiation into dendritic cells, leading to the establishment of a prometastatic niche, through the production of IL- $6^{66}$. One of the components of tumor derived ECVs is represented by miRNAs. Circulating miRNAs are a highly heterogeneous compartment of the circulating RNA and their evaluation is quite difficult in plasma but, on the counterpart, they are enriched in ECVs. This has been demonstrated on a mouse model of orthotopic xenografts from a breast tumor, showing a high concentration of miR-1246 in ECVs, and confirmed in vesicles isolated from breast cancer patients, correlating with patients' likelihood of developing recurrencies of the disease ${ }^{67}$. Many other circulating miRNAs and in general noncoding RNAs have been correlated with breast cancer prognosis, miR-34a being significantly downregulated in breast cancer compared to healthy controls ${ }^{68-71}$.

The other abundant tumor-derived circulating component derived from a liquid biopsy is represented by the ctDNA. In the specific case of breast cancer, ctDNA has 
been correlated to patients' relapse after surgery and adjuvant treatment, predicting it one to two years before the clinical evidence of the metastatic disease, also correlating with the disease burden ${ }^{72}$. Moreover, ctDNA has been shown to correlate to tumor dynamics in breast cancer patients undergoing surgical or medical therapy ${ }^{73}$, and to be a valuable screening methodology for the selection of breast cancer patients for a specific target drug treatment ${ }^{74,75}$.

Some methodological limitations are still embedded in the liquid biopsies per se. The elements that can be analyzed form a liquid biopsy, especially in the case of ctDNA, still need to be paired to the genomic sequencing of the primary tumor. ctDNA sequencing relies on small circulating fragments which are very diluted among the normal circulating DNA, and therefore represent an impure source of information. Furthermore, in the case of a metastatic disease it is still difficult to assess polyclonal versus monoclonal seeding due to the still ineffective bioinformatics tools available ${ }^{76}$.

\section{Liquid biopsies in neuroblastoma}

Despite relatively rare neuroblastoma (NB) represents a significant clinical problem at pediatric age, accounting for a significant fraction (approximately 10\%) of death from children malignancies. Originated from sympathetic nervous system, NB represents the most frequent extracranial tumor in children. Stratification of patients can be conducted on the basis of age at the diagnosis, International Neuroblastoma Staging System (INSS) stage, the MYCN status, Shimada histopathology, and ploidy, defining three well discriminated groups of low, intermediated, and high risk ${ }^{77}$. Genomic abnormalities have indeed been sufficiently defined and linked to the clinical presentation; deletions on chromosomes $1 \mathrm{p}, 11 \mathrm{q}$, or gains on 17q2,3 are examples of effective prognostic markers of the clinical outcome, despite the molecular basis of their contribution to the pathogenesis are not entirely clear $^{78-81}$. Moreover, neuronal differentiation markers such as p73 have also been associated to the pathogenesis of the disease ${ }^{82-84}$, while the amplification of the protooncogene MYCN is another clear predictor of disease severity, therapy resistance and poor clinical outcome. Less than $50 \%$ of NB patients with MYCN amplification reach 5 -year survival, on contrary of the $90 \%$ of non-MYC patients $^{21,64,85,86}$.

Diagnostic procedures for NB largely rely on radiographic body imaging, nuclear medicine examination, evaluation of serum levels of neuron-specific enzymes (enolase and lactate dehydrogenase) or urinary levels of vanillylmandelic acid (VMA) and homovanillic acid (HVA). Sensitivity of these techniques is however low to represent proper early detection methods. Their diagnostic value emerges indeed quite late when the cancer has developed into clinical stage and discrete lumps evident.

Detection of cell free DNA (cfDNA) for NB could improve effectiveness of diagnostic methods and tolerability of medical procedure especially at young age of the patients which are generally involved in this. Detection of MYCN DNA by PCR in the peripheral blood was firstly reported in 2002. Those early data indicated that data the release of MYCN DNA in the blood circulation is an early process in disease, thus offering a potential novel marker for patient follow-up after treatment ${ }^{87}$. Further refinement studies introduced NAGK as a reference gene to quantify MYCN copy number as an MYCN/NAGK (M/ $\mathrm{N})$ ratio, providing a more accurate assessment of $\mathrm{MYCN}$ status $^{88}$. Subsequent studies have however reduced the optimism, as sensitivity on patients with NB of INSS stages 1 and 2 appeared to be significantly lower than the core biopsy analyses ${ }^{89,90}$. Another putative biomarker is the serum detection of ALK DNA. ALK is a tyrosine kinase receptor subjected to copy number amplification in $25 \%$ of NB cases, and some instance mutations in ALK sequence have also been observed. Detection of ALK circulating DNA has therefore been explored to achieve approaches to monitor relapse and predict drug resistance. For example, F1174 and R1275 mutations appear to be effectively detected with high specificity and sensitivity in the peripheral blood of a cohort of NB patients ${ }^{91}$. Additional effort has been placed in development of methods to detect chromosomal variations and DNA methylation in liquid biopsies from NB patients. Good concordance between primary tumor and liquid biopsies has been revealed in detection of several chromosomal variations (11q, 17q, and $1 \mathrm{p})$, it is however still missing a reliable large-scale clinical trial reporting substantial evidence to support shift of diagnostic procedures to detection of cfDNA. At the present, clinical practise therefore relies on histopathology and molecular characterization of tumor tissue ${ }^{63,92,93}$. However, unfeasibility of multiple resections, irregular interval between morbidity and acquisition of diagnostic material and ambiguous interpretations of pathology samples are just some of the substantial drawbacks of these classic procedures.

\section{Conclusions}

Oncology has undoubtedly entered the precision medicine era with the technological ability to characterize a single cancer from multiomics approaches. This possibility can be exploited also thanks to some major advances in artificial intelligence algorithms, that are essential for deciphering much of the information embedded in the omics data. Anyhow, this huge amount of biologic details does not readily and dynamically translate into the clinical practice, since it is usually performed on the cancer tissue itself, that is normally taken one (or a very few times) 
during individual cancer history. This limitation does not only regard time, but also capturing tumor heterogeneity. The improvement of our understanding of cancer biology, with the discovery that tumoral material circulates in the bloodstream in form of CTCs, ctDNA, or ctRNA, EVCs, has given the opportunity to conceive the idea that blood itself might represent a cancer biopsy per se. Under this light, liquid biopsies can overcome many limitations of tissue biopsies and could capture tumor heterogeneity in a whole, thanks to the improvement of both omics technologies and the associated artificial intelligence elaboration of the data, but mostly can capture tumor evolution without being invasive to the patients. This will be soon translated into a more precise prognostic evaluation and a best treatment selection designed on a single patient's disease during its evolution, leading to a true precision medicine approach.

\section{Acknowledgements}

This work has been supported by the Associazione Italiana per la Ricerca contro il Cancro (AIRC) to G.M. (IG\#20473; 2018-2022), to I.A. (AIRC Start-Up ID 23219; 2020-2024), to E.C. (IG\#22206; 2019-2023), Ministry of Health \& MAECI Italy-China Science and Technology Cooperation (\#PGR00961) to G.M. and Y.W.

\section{Author details}

${ }^{1}$ Torvergata Oncoscience Research Centre of Excellence, TOR, Department of Experimental Medicine, University of Rome Tor Vergata, 00133 Rome, Italy.

${ }^{2}$ School of Life Sciences, University of Nottingham, Nottingham, UK. ${ }^{3}$ San Carlo di Nancy Hospital, Rome, Italy. ${ }^{4}$ Indivumed GmbH, Hamburg, Germany. ${ }^{5}$ Medstar Georgetown University Hospital, Georgetown University, Washington, DC, USA. ${ }^{6}$ CAS Key Laboratory of Tissue Microenvironment and Tumor, Shanghai Institute of Nutrition and Health, Shanghai Institutes for Biological Sciences, University of Chinese Academy of Sciences, Chinese Academy of Sciences, 320 Yueyang Road, 200031 Shanghai, China. ${ }^{7}$ The First Affiliated Hospital of Soochow University and State Key Laboratory of Radiation Medicine and Protection, Institutes for Translational Medicine, Soochow University, 199 Renai Road, 215123 Suzhou, Jiangsu, China

\section{Author contributions}

I.A., G.M., and C.G. wrote the manuscript. CG prepared the figures. All the other indicated authors (R.B., P.B., E.C., M.C., C.C., N.D.D., H.J., A.M., C.M., J.M., M.M., G.P., M.P., G.S., M.T., V.R., G.T., Y.S., and Y.W.) made substantial contribution to the conception of the manuscript and critically revised it. All of the Authors have approved this submitted version.

\section{Ethics approval and consent to participate}

Not applicable.

\section{Conflict of interest}

The authors declare that they have no conflict of interest.

\section{Publisher's note}

Springer Nature remains neutral with regard to jurisdictional claims in published maps and institutional affiliations.

Received: 29 October 2020 Revised: 3 November 2020 Accepted: 5 November 2020

Published online: 26 November 2020

\footnotetext{
References

1. Rodriguez-Martin, B. et al. Pan-cancer analysis of whole genomes identifies driver rearrangements promoted by LINE-1 retrotransposition. Nat. Genet. 52, 306-319 (2020)
}

2. Lamberti, G. et al. Beyond EGFR, ALK and ROS1: current evidence and future perspectives on newly targetable oncogenic drivers in lung adenocarcinoma. Crit. Rev. Oncol. Hematol. 156, 103119 (2020).

3. Jin, Z. \& Sinicrope, F. A. Advances in the therapy of BRAF(V600E) metastatic colorectal cancer. Expert Rev. Anticancer Ther. 19, 823-829 (2019).

4. D'Adamo, G. L., Widdop, J. T. \& Giles, E. M. The future is now? Clinical and translational aspects of "Omics" technologies. Immunol. Cell Biol. https:/doi. org/10.1111/imcb.12404 (2020).

5. Jameson, J. L. \& Longo, D. L. Precision medicine-personalized, problematic, and promising. N. Engl. J. Med. 372, 2229-2234 (2015).

6. Mirnezami, R., Nicholson, J. \& Darzi, A. Preparing for precision medicine. N. Engl. J. Med. 366, 489-491 (2012).

7. Polley, M. C., Korn, E. L. \& Freidlin, B. Phase III precision medicine clinical trial designs that integrate treatment and biomarker evaluation. JCO Precis. Oncol. https://doi.org/10.1200/po.18.00416 (2019).

8. Mittal, K. et al. Dual VEGFNEGFR inhibition in advanced solid malignancies: clinical effects and pharmacodynamic biomarkers. Cancer Biol. Ther. 15, 975-981 (2014).

9. Habeck, M. FDA licences imatinib mesylate for CML. Lancet Oncol. 3, 6 (2002).

10. Heng, D. Y. et al. Primary anti-vascular endothelial growth factor (VEGF)refractory metastatic renal cell carcinoma: clinical characteristics, risk factors, and subsequent therapy. Ann. Oncol. 23, 1549-1555 (2012).

11. Gerlinger, M. et al. Intratumor heterogeneity and branched evolution revealed by multiregion sequencing. N. Engl. J. Med 366, 883-892 (2012).

12. Gerlinger, M. \& Swanton, C. How Darwinian models inform therapeutic failure initiated by clonal heterogeneity in cancer medicine. Br. J. Cancer 103, 1139-1143 (2010)

13. Gerlinger, T. \& Urban, H. J. From heterogeneity to harmonization? Recent trends in European health policy. Cad. Saude Publica 23, S133-S142 (2007).

14. Yap, T. A., Gerlinger, M., Futreal, P. A., Pusztai, L. \& Swanton, C. Intratumor heterogeneity: seeing the wood for the trees. Sci. Transl. Med. 4, 127ps110 (2012).

15. Mihaylov, I., Kańduła, M., Krachunov, M. \& Vassilev, D. A novel framework for horizontal and vertical data integration in cancer studies with application to survival time prediction models. Biol. Direct 14, 22-22 (2019).

16. Jiao, W. et al. A deep learning system accurately classifies primary and metastatic cancers using passenger mutation patterns. Nat. Commun. 11, 728-728 (2020).

17. Li, C. et al. Integrated omics of metastatic colorectal cancer. Cancer Cell https:// doi.org/10.1016/j.ccell.2020.08.002 (2020).

18. Pcawg Transcriptome Core Group, C. et al. Genomic basis for RNA alterations in cancer. Nature 578, 129-136 (2020).

19. Wise, J. F. \& Lawrence, M. S. Genomes captured during tumour spread. Nature 575, 60-61 (2019).

20. Kim, S. Y., Jeong, H.-H., Kim, J., Moon, J.-H. \& Sohn, K.-A. Robust pathway-based multi-omics data integration using directed random walks for survival prediction in multiple cancer studies. Biol. Direct 14, 8-8 (2019).

21. Hidalgo, M. R., Amadoz, A., Çubuk, C., Carbonell-Caballero, J. \& Dopazo, J. Models of cell signaling uncover molecular mechanisms of high-risk neuroblastoma and predict disease outcome. Biol. Direct 13, 16-16 (2018).

22. Liu, L. et al. Computational identification and characterization of glioma candidate biomarkers through multi-omics integrative profiling. Biol. Direct 15, 10 (2020).

23. Dobon, B., Montanucci, L., Pereto, J., Bertranpetit, J. \& Laayouni, H. Gene connectivity and enzyme evolution in the human metabolic network. Biol. Direct 14, 17 (2019).

24. Panchin, A. Y., Aleoshin, V. V. \& Panchin, Y. V. From tumors to species: a SCANDAL hypothesis. Biol. direct 14, 3-3 (2019).

25. Interno, V. et al. Liquid biopsy as a tool exploring in real-time both genomic perturbation and resistance to EGFR antagonists in colorectal cancer. Front. Oncol. 10, 581130 (2020).

26. Indraccolo, S. Evolving use of liquid biopsy in non-small-cell-lung cancer patients. Int. J. Biol. Markers 35, 23-25 (2020).

27. Garcia, C. M. \& Toms, S. A. The role of circulating MicroRNA in glioblastoma liquid biopsy. World Neurosurg. 138, 425-435 (2020).

28. Fu, Y., Zhang, Y. \& Khoo, B. L. Liquid biopsy technologies for hematological diseases. Med. Res. Rev. https://doi.org/10.1002/med.21731 (2020).

29. Diehl, F. et al. Detection and quantification of mutations in the plasma of patients with colorectal tumors. Proc. Natl Acad. Sci. USA 102, 16368-16373 (2005).

30. Dominguez-Vigil, I. G., Moreno-Martinez, A. K., Wang, J. Y., Roehrl, M. H. A. \& Barrera-Saldana, H. A. The dawn of the liquid biopsy in the fight against cancer. Oncotarget 9, 2912-2922 (2018). 
31. Riethdorf, S., O'Flaherty, L., Hille, C. \& Pantel, K. Clinical applications of the CellSearch platform in cancer patients. Adv. Drug Deliv. Rev. 125, 102-121 (2018).

32. Wang, L. et al. Promise and limits of the CellSearch platform for evaluating pharmacodynamics in circulating tumor cells. Semin. Oncol. 43, 464-475 (2016).

33. Praharaj, P. P., Bhutia, S. K., Nagrath, S., Bitting, R. L. \& Deep, G. Circulating tumor cell-derived organoids: current challenges and promises in medical research and precision medicine. Biochim. Biophys. Acta Rev. Cancer 1869, 117-127 (2018).

34. Wang, H. X. \& Gires, O. Tumor-derived extracellular vesicles in breast cancer: from bench to bedside. Cancer Lett. 460, 54-64 (2019).

35. Ozawa, P. M. M. et al. Liquid biopsy for breast cancer using extracellular vesicles and cell-free microRNAs as biomarkers. Transl. Res. 223, 40-60 (2020).

36. Peng, M., Chen, C., Hulbert, A., Brock, M. V. \& Yu, F. Non-blood circulating tumor DNA detection in cancer. Oncotarget 8, 69162-69173 (2017).

37. Caldas, C. et al. Detection of K-ras mutations in the stool of patients with pancreatic adenocarcinoma and pancreatic ductal hyperplasia. Cancer Res. 54, 3568-3573 (1994).

38. Kisiel, J. B. et al. Stool DNA testing for the detection of pancreatic cancer: assessment of methylation marker candidates. Cancer 118, 2623-2631 (2012).

39. Laugsand, E. A., Brenne, S. S. \& Skorpen, F. DNA methylation markers detected in blood, stool, urine, and tissue in colorectal cancer: a systematic review of paired samples. Int. J. Colorectal Dis. https://doi.org/10.1007/s00384-020-03757$x(2020)$.

40. Ponti, G., Manfredini, M. \& Tomasi, A. Non-blood sources of cell-free DNA for cancer molecular profiling in clinical pathology and oncology. Crit. Rev. Oncol. Hematol. 141, 36-42 (2019).

41. Langheinrich, M. et al. Microbiome patterns in matched bile, duodenal, pancreatic tumor tissue, drainage, and stool samples: association with preoperative stenting and postoperative pancreatic fistula development. J. Clin. Med. https://doi.org/10.3390/jcm9092785 (2020).

42. Biancone, L. et al. Non-invasive techniques for assessing postoperative recurrence in Crohn's disease. Dig. Liver Dis. 40, S265-S270 (2008).

43. Sileri, $\mathrm{P}$. et al. Ischemic preconditioning protects intestine from prolonged ischemia. Transpl. Proc. 36, 283-285 (2004)

44. Caputo, A., Fournier, P.E. \& Raoult, D. Genome and pan-genome analysis to classify emerging bacteria. Biol. Direct 14, 5-5 (2019).

45. Gerner, S. M., Rattei, T. \& Graf, A. B. Assessment of urban microbiome assemblies with the help of targeted in silico gold standards. Biol. Direct 13, 22-22 (2018).

46. Kawulok, J., Kawulok, M. \& Deorowicz, S. Environmental metagenome classification for constructing a microbiome fingerprint. Biol. Direct 14, $20-20$ (2019).

47. Osmanovic, D., Kessler, D. A., Rabin, Y. \& Soen, Y. Darwinian selection of host and bacteria supports emergence of Lamarckian-like adaptation of the system as a whole. Biol. Direct 13, 24-24 (2018).

48. Clos-Garcia, M. et al. Integrative analysis of fecal metagenomics and metabolomics in colorectal cancer. Cancers https://doi.org/10.3390/cancers12051142 (2020).

49. Casimiro-Soriguer, C. S., Loucera, C., Perez Florido, J., López-López, D. \& Dopazo, J. Antibiotic resistance and metabolic profiles as functional biomarkers that accurately predict the geographic origin of city metagenomics samples. Biol. Direct 14, 15-15 (2019).

50. Ryan, F. J. Application of machine learning techniques for creating urban microbial fingerprints. Biol. Direct 14, 13-13 (2019).

51. Walker, A. R. \& Datta, S. Identification of city specific important bacterial signature for the MetaSUB CAMDA challenge microbiome data. Biol. Direct 14, 11-11 (2019).

52. Walker, A. R., Grimes, T. L., Datta, S. \& Datta, S. Unraveling bacterial fingerprints of city subways from microbiome 165 gene profiles. Biol. Direct 13, 10-10 (2018).

53. Zhu, C. et al. Fingerprinting cities: differentiating subway microbiome functionality. Biol. Direct 14, 19-19 (2019).

54. Zolfo, M. et al. Profiling microbial strains in urban environments using metagenomic sequencing data. Biol. Direct 13, 9-9 (2018).

55. Ivanova, E., Ward, A., Wiegmans, A. P. \& Richard, D. J. Circulating tumor cells in metastatic breast cancer: from genome instability to metastasis. Front. Mol. Biosci. 7, 134 (2020).

56. Mego, M., Mani, S. A. \& Cristofanilli, M. Molecular mechanisms of metastasis in breast cancer-clinical applications. Nat. Rev. Clin. Oncol. 7, 693-701 (2010).

57. Agelaki, S. et al. Phenotypic characterization of circulating tumor cells in triple negative breast cancer patients. Oncotarget 8, 5309-5322 (2017).
58. Yu, M. et al. Circulating breast tumor cells exhibit dynamic changes in epithelial and mesenchymal composition. Science 339, 580-584 (2013).

59. Bill, R. \& Christofori, G. The relevance of EMT in breast cancer metastasis: Correlation or causality? FEBS Lett. 589, 1577-1587 (2015).

60. Boral, D. et al. Molecular characterization of breast cancer CTCs associated with brain metastasis. Nat. Commun. 8, 196 (2017).

61. Ankney, J. A., Xie, L., Wrobel, J. A., Wang, L. \& Chen, X. Novel secretome-totranscriptome integrated or secreto-transcriptomic approach to reveal liquid biopsy biomarkers for predicting individualized prognosis of breast cancer patients. BMC Med. Genomics 12, 78 (2019).

62. Whelan, S. A. et al. Mass spectrometry (LC-MS/MS) identified proteomic biosignatures of breast cancer in proximal fluid. J. Proteome Res. 11, 5034-5045 (2012).

63. Han, Y. et al. Integration of molecular features with clinical information for predicting outcomes for neuroblastoma patients. Biol. Direct 14, 16-16 (2019).

64. Grimes, T., Walker, A. R., Datta, S. \& Datta, S. Predicting survival times for neuroblastoma patients using RNA-seq expression profiles. Biol. Direct 13, 11-11 (2018).

65. Mertins, P. et al. Proteogenomics connects somatic mutations to signalling in breast cancer. Nature 534, 55-62 (2016).

66. $\mathrm{Yu}, \mathrm{S}$. et al. Tumor exosomes inhibit differentiation of bone marrow dendritic cells. J. Immunol. 178, 6867-6875 (2007).

67. Hannafon, B. N. et al. Plasma exosome microRNAs are indicative of breast cancer. Breast Cancer Res. 18, 90 (2016).

68. Agostini, M. \& Knight, R. A. miR-34: from bench to bedside. Oncotarget 5, 872-881 (2014).

69. Agostini, M., Ganini, C., Candi, E. \& Melino, G. The role of noncoding RNAs in epithelial cancer. Cell Death Discov. 6, 13 (2020).

70. Wei, R. et al. Liquid biopsy of extracellular vesicle-derived miR-193a-5p in colorectal cancer and discovery of its tumor-suppressor functions. Front. Oncol. 10, 1372 (2020).

71. Wang, L. et al. Long non-coding RNA LINC00426 contributes to doxorubicin resistance by sponging miR-4319 in osteosarcoma. Biol. Direct 15, 11 (2020).

72. Coombes, R. C. et al. Personalized detection of circulating tumor DNA antedates breast cancer metastatic recurrence. Clin. Cancer Res. 25, 4255-4263 (2019).

73. Diehl, F. et al. Circulating mutant DNA to assess tumor dynamics. Nat. Med. 14, 985-990 (2008).

74. Ma, C. X. et al. Neratinib efficacy and circulating tumor DNA detection of HER2 mutations in HER2 nonamplified metastatic breast cancer. Clin. Cancer Res. 23, 5687-5695 (2017).

75. Oktay, K. et al. A computational statistics approach to evaluate blood biomarkers for breast cancer risk stratification. horm. Cancer 11, 17-33 (2020).

76. Cresswell, G. D. et al. Mapping the breast cancer metastatic cascade onto ctDNA using genetic and epigenetic clonal tracking. Nat. Commun. 11, 1446 (2020).

77. Francescatto, $M$. et al. Multiomics integration for neuroblastoma clinical endpoint prediction. Biol. Direct 13, 5-5 (2018).

78. Nicolai, S., Pieraccioli, M., Peschiaroli, A., Melino, G. \& Raschellà, G. Neuroblastoma: oncogenic mechanisms and therapeutic exploitation of necroptosis. Cell Death Dis. 6, e2010-e2010 (2015).

79. Polewko-Klim, A., Lesiński, W., Mnich, K., Piliszek, R. \& Rudnicki, W. R. Integration of multiple types of genetic markers for neuroblastoma may contribute to improved prediction of the overall survival. Biol. Direct 13, 17-17 (2018).

80. Suo, C. et al. Accumulation of potential driver genes with genomic alterations predicts survival of high-risk neuroblastoma patients. Biol. Direct 13, 14-14 (2018).

81. Pieraccioli, M. et al. ZNF281 inhibits neuronal differentiation and is a prognostic marker for neuroblastoma. Proc. Natl Acad. Sci. USA 115, 7356-7361 (2018).

82. Tomasini, R. et al. TAp73 knockout shows genomic instability with infertility and tumor suppressor functions. Genes Dev. 22, 2677-2691 (2008).

83. Wilhelm, M. T. et al. Isoform-specific p73 knockout mice reveal a novel role for delta Np73 in the DNA damage response pathway. Genes Dev. 24, 549-560 (2010).

84. Agostini, M. et al. Metabolic reprogramming during neuronal differentiation. Cell Death Differ. 23, 1502-1514 (2016).

85. Amelio, I. et al. SynTarget: an online tool to test the synergetic effect of genes on survival outcome in cancer. Cell Death Differ. 23, 912 (2016).

86. Han, Y. et al. Integrative analysis based on survival associated co-expression gene modules for predicting Neuroblastoma patients' survival time. Biol. Direct 14, 4-4 (2019).

87. Combaret, V. et al. Circulating MYCN DNA as a tumor-specific marker in neuroblastoma patients. Cancer Res. 62, 3646-3648 (2002). 
88. Gotoh, T. et al. Prediction of MYCN amplification in neuroblastoma using serum DNA and real-time quantitative polymerase chain reaction. J. Clin. Oncol. 23, 5205-5210 (2005).

89. Yagyu, S. et al. Serum-based quantification of MYCN gene amplification in young patients with neuroblastoma: potential utility as a surrogate biomarker for neuroblastoma. PLoS ONE 11, e0161039 (2016).

90. Combaret, V. et al. Influence of neuroblastoma stage on serum-based detection of MYCN amplification. Pediatr. Blood Cancer 53, 329-331 (2009).
91. Combaret, V. et al. Detection of tumor ALK status in neuroblastoma patients using peripheral blood. Cancer Med. 4, 540-550 (2015).

92. Baali, I., Acar, D. A. E., Aderinwale, T. W., HafezQorani, S. \& Kazan, H. Predicting clinical outcomes in neuroblastoma with genomic data integration. Biol. Direct 13, 20-20 (2018).

93. Tranchevent, L.-C. et al. Predicting clinical outcome of neuroblastoma patients using an integrative network-based approach. Biol. direct 13, 12-12 (2018). 